\title{
Improve quality and productivity of some faba bean cultivars with foliar application of fulvic acid
}

\author{
Y. R. Abdel-Baky ${ }^{1 *}$, H. F. Abouziena' ${ }^{1}$ A. A. Amin', M. Rashad El-Sh ${ }^{1}$ and A. M. Abd El-Sttar ${ }^{2}$
}

\begin{abstract}
Background: Two field experiments were carried out at the Research and Production Station of the National Research Centre, Nubaria region, Behira Governorate, Egypt, during two winter seasons of 2014/2015 and 2015/2016. The aim of this study was to investigate the response of vegetative growth, yield, yield components, and some metabolic constituents of four faba bean (Vicia faba L.) cultivars "Nubaria 2, Sakha 1, Sakha 3, and Sakha 4" to foliar application of fulvic acid (FA) at 0.0, 3, 6, and $9 \mathrm{gL}^{-1}$.

Results: The results show that all applications of FA increased all vegetative characters (i.e., plant height, number of branches and leaves, and total dry weight/plant, fourth leaf area and leaf area index, specific leaf weight, and crop growth rate), as well as, yield and its components (i.e., number of pods/plant, weight of pods/plant, seed and straw yield/plant, seed and straw yield/feddan, and seed index) of all studied faba bean cultivars, comparing to untreated plants. The results also show the total photosynthetic pigments content in leaves were increased by increasing FA concentration up to $9 \mathrm{gL}^{-1}$. Treatment $\mathrm{FA}$ at $9 \mathrm{gL}^{-1}$, significantly improved the nutritional value and quality of seeds by increasing total carbohydrates, crude protein, minerals (nitrogen, phosphorus, and potassium) content, arginine, lysine, phenylalanine, and tryptophan.
\end{abstract}

Conclusions: Generally, foliar application of FA at $9 \mathrm{gL}^{-1}$, singly or interaction with Sakha 4 effectively increased seed yield and quality. In conclusion, the growth and yield contributing characters and quality of four bean cultivars could be improved with foliar application of FA treatments.

Keywords: Faba bean, Fulvic acid, FA, Photosynthetic pigments, Yield, Biochemical constituents, Cultivars

\section{Introduction}

Faba bean (Vicia faba L.) family (Fabaceae) is one of the most important legume crops, providing between 28 and $30 \%$ of the dietary protein for humans, $51-68 \%$ total carbohydrates, and considered as a good source of natural antioxidants (Chaieb et al. 2011). Faba bean is one of the major crops that consumed worldwide for feeding farm animals (Cazzato et al. 2012) and their seeds are high in lysine and arginine, which may complement the low levels in cereals. Faba bean increases humus of soil and use in crop rotation (Kumar et al. 2015).

The use of bioeffectors formally known as plant biostimulants or agricultural biostimulants, has become common a

\footnotetext{
*Correspondence: yrefaai@yahoo.com

'Department of Botany, National Research Centre, El Buhouth St., Dokki, Cairo 12622, Egypt

Full list of author information is available at the end of the article
}

practice in agriculture and provides a number of benefits in stimulating growth and protecting against stresses. A biostimulant is loosely defined as an organic material and/or microorganism that is applied to enhance nutrient uptake, reduce fertilizers' consumption, stimulate growth, and enhance stress tolerance or crop quality (Van Oosten et al. 2017). Humic substances (HSs) are the major natural component of soil organic matter formed in soil from the decomposition of dead cell materials by microorganisms (Nardi et al. 2007). Humic substances include humic acids, fulvic acids, and humins (Berbara and García 2014). HSs have indirect effects (increase in fertilizer efficiency or reducing soil compaction) or direct (improvement in the overall plant biomass) effects on plant growth. The use of humic substances can increase the root growth and uptake of some nutrients such as $\mathrm{N}, \mathrm{Fe}, \mathrm{P}, \mathrm{K}, \mathrm{Ca}$, $\mathrm{Zn}$, and $\mathrm{Mg}$ and enhance tolerance to abiotic stress 
such as salinity. The variability in effects of HSs is due to the source of the HSs, the environmental conditions, the receiving plant, and the dose and manner of HS application (Rose et al. 2014).

Fulvic acids (FAs) are humic acids with a higher oxygen content and lower molecular weight ranging only to a few hundred Daltons and can pass through micropores of biological or artificial membrane systems, while humic acids cannot, with larger molecule weights ranging to a few thousand Daltons (Bulgari et al. 2015). Fulvic acids have greater total acidity, greater numbers of carboxyl groups, and higher adsorption and cation exchange capacities than humic acid and may play roles as natural chelators in the mobilization and transport of micronutrients (Bocanegra et al. 2006). Fulvic acids can remain in soil solution even at high salt concentrations and at a wide range of $\mathrm{pH}$ (Zimmerli et al. 2008) and have long-lasting potential to interact with plant roots. Fulvic acid is considered to be the soil organic fraction that is soluble in both acid and alkali. Fulvic acid promotes some physiological processes depending on plant species, developmental stage, and application conditions, e.g., soil applications of a "humic substance" that was shown with analysis to be $90.7 \%$ fulvic acid, increased fruit weight, fruit equatorial diameter, juice $\mathrm{pH}$, and vitamin $\mathrm{C}$ content of lemon (Citrus limon) trees, while foliar applications of FA increased yield of maize under drought conditions (Anjum et al. 2011). Further studies with pepper, showed that FA applied as drenches, enhanced multiple parameters of fruit quality and antioxidant activity (Aminifard et al. 2012).

The potent impact of humic substances on various areas of plant structure and function has prompted many investigators to apply them to several crop plants aiming to control growth patterns and development. In common bean, FA enhanced the numbers of root initials on hypocotyl sections, increased root elongation of maize (Eyheraguibel et al. 2008), number and length of lateral roots of both Arabidopsis and tomato (Dobbss et al. 2007), dry weight of shoot of maize (Anjum et al. 2011), and number of flowers per cucumber plant (Rauthan and Schnitzer 1981). Fulvic acids enhanced the uptake of ${ }^{32} \mathrm{P}$ phosphate in beech and $\mathrm{N}$ content in maize plants (Eyheraguibel et al. 2008), increased chlorophyll content in both soybean and ryegrass (Chen et al. 2004), photosynthesis rate in maize (Anjum et al. 2011), intercellular levels of ATP, glucose-6-phosphate, and growth promotion in cell cultures of Greek fir (Zancani et al. 2011). Moreover, humic substances enhanced the expression of the phenylalanine (tyrosine) ammonia-lyase (PAL/TAL) that catalysis the first main step in the biosynthesis of phenolics and provides an approach to plant responses to stress (Schiavon et al. 2010). Humates significantly enhanced the activity of PAL in tomato leaves (Olivares et al. 2015).
Thus, fulvic acid is expected to influence the growth and yield of faba bean cultivars. Therefore, the present investigation was undertaken to study the impact of spraying different concentrations of FA on four faba bean cultivars (Noubaria 2, Sakha 1, Sakha 3, and Sakha 4) on some morphological criteria, yield, and some metabolic constituents of their seeds in a bid to find suitable concentration and cultivar that could enhance yield.

\section{Materials and methods}

Two field experiments were carried out at the Research and Production Station of the National Research Centre, Nubaria region, Behira Governorate, Egypt, during two successive seasons of winter 2014/2015 and 2015/2016. Seeds of four faba bean cultivars (Vicia faba L.; cv. Noubaria 2, Sakha 1, Sakha 3, and Sakha 4) were sown on 15th November in both seasons in sandy soil. The physical and chemical proprieties of the soil are presented in Table 1 according to Chapman and Pratt (1978). The experimental design was a split plot with four replications. The cultivars occupied the main plots, and the fulvic acid treatments were allocated at random in subplots. The plot area was $10.5 \mathrm{~m}^{2}(3.0 \mathrm{~m} \times 3.5 \mathrm{~m})$ and consisted of five ridges $70 \mathrm{~cm}$ apart; seeds were planted at the rate of $70 \mathrm{~kg} /$ feddan using the dry planting on the two sides of the ridge in hills the distance between hills was $25 \mathrm{~cm}$. Calcium superphosphate $\left(15.5 \% \mathrm{P}_{2} \mathrm{O}_{5}\right)$ was added presowing at $200 \mathrm{~kg} / \mathrm{fed}$. to the soil; similarly, nitrogen in the form of ammonium nitrate $(33.0 \% \mathrm{~N})$ was applied at the rate of $100 \mathrm{kgN} / \mathrm{fed}$. as start dose before the first irrigation. Potassium sulphate $\left(48 \% \mathrm{~K}_{2} \mathrm{O}\right)$ was added at the rate of $30 \mathrm{~kg} / \mathrm{fed}$. to the soil during seed bed preparation.

Faba bean plants were irrigated and maintained during the whole growth season up to $100 \%$ of potential crop evapotranspiration (in 15 successive irrigations) using sprinkler irrigation system. The good agricultural practices, pest control, for growing faba bean in sandy soil were applied as recommended. In both seasons, a foliar spray was applied twice to faba bean plants during

Table 1 Soil mechanical and chemical analysis characters

\begin{tabular}{|c|c|c|c|}
\hline Character & Value & Character & Value \\
\hline Sand $\%$ & 88 & K & 10.18 \\
\hline Silt \% & 4 & Ca mg/100 g & 92.0 \\
\hline Clay \% & 7.2 & $\mathrm{Mg}$ & 18.4 \\
\hline Texture & Sandy & $\mathrm{Na}$ & 12.36 \\
\hline $\mathrm{pH}$ (1: 2.5 water) & 8.83 & $\mathrm{Fe}$ & 8.92 \\
\hline E.C $(\mathrm{mmhos} / \mathrm{cm})(1: 2.5)$ & 0.12 & $\mathrm{Mn} \mathrm{mg} / \mathrm{kg}$ & 8.34 \\
\hline $\mathrm{CaCO}_{3} \%$ & 4.8 & $\mathrm{Zn}$ & 0.13 \\
\hline O.M \% & 0.24 & $\mathrm{Cu}$ & 0.10 \\
\hline$P$ & 0.22 & & \\
\hline
\end{tabular}


elongation stage (at 45 and 60 days after sowing (DAS)), with fulvic acid $\left(\mathrm{C}_{14} \mathrm{H}_{12} \mathrm{O}_{8}\right)$, it is sourced from Sigma Aldrich Co. (FA; 0.0, 3, 6, and $9 \mathrm{gL}^{-1}$ ), to four bean cultivars in addition control plants which were sprayed with tap water.

Plant growth characters measured at 75, 90, and 105 (green yield) DAS, by random samples of ten plants were taken from each treatment, to determine plant height $(\mathrm{PH})$, branch number $(\mathrm{BN})$, leaf number (LN), total dry weight/plant (TDW), fourth leaf area (LA) $\left(\mathrm{cm}^{2} /\right.$ plant) by leaf disc method on dry weight basis according to Vivekanadan et al. (1972), leaf area index (LAI) according to Sestak et al. (1971), specific leaf weight (SLW) $\left(\mathrm{mg} / \mathrm{cm}^{2}\right)$ according to Radford (1967), and crop growth rate (CGR) $\left(\mathrm{mg} / \mathrm{cm}^{2} /\right.$ days) according to Abdel-Gawad et al. (1980). The photosynthetic pigment $[(\mathrm{Chl}) a, b$, and carotenoids as well as total photosynthetic pigments (TPP)] content of fresh leaves was also determined at 75, 90, and 105 DAS according to (Lichtenthaler and Wellburn 1983).

At the time of harvest (115 DAS), the mean values of yield and its related parameters [i.e., plant height (PH), branch number $(\mathrm{BN})$, number of pods/plant $(\mathrm{PN} / \mathrm{P})$, weight of pods/plant (PW/P), seed index (SI), seed and straw yields/plant (SY/P; StY/P), and seed and straw yields/fed (SY/F; StY/F)] were determined.

Seeds were harvested from each treatment and dried in an electric oven with a drift fan at $70^{\circ} \mathrm{C}$ for $48 \mathrm{~h}$ until constant dry weight was achieved. Dry samples of seeds were used to total carbohydrate percent in seeds (CP) according to (Dubois et al. 1956). The method of Plummer (1978) was used to estimate total free amino acids (FAA). The dried powder samples were wet digested as described in the method of Chapman and Pratt (1978). The acid digest of the plant matter was analyzed for determination of nitrogen, phosphorus, and potassium according to the following methods: Total nitrogen was determined using the modified Micro-Kjeldahl method. Crude protein percentage (CPP) was calculated by multiplying the values of total $\mathrm{N}$ by 6.25 (A.O.A.C. 1988). Phosphorus was determined by atomic absorption spectrophotometer according to Carpena et al. (1990). Potassium was measured using flame photometer (atomic spectra AAS vario 6) according to Brown and Lilliland (1946). Mineral contents were expressed in milligram $100 \mathrm{~g}^{-1}$ DW seed tissue.

Data for both growing seasons were measured according to Snedecor and Cochran (1990). Data were combined because the coefficient of variation (CV\%) for each season's data was $<5 \%$. Data were analyzed by analysis of variance (ANOVA) for the split-plot design, and treatment means were compared using Fisher's least significant difference (LSD) at $\alpha=0.05$, using means of "MSTAT-C" computer software package.

\section{Results}

\section{Growth parameters}

Data presented in Tables 1 and 2 show that foliar application of FA either separately at 3, 6, and $9 \mathrm{gL}^{-1}$ and four faba bean cultivars "Noubaria 2, Sakha 1, Sakha 3, and Sakha 4" or their interaction, promoted almost all growth criteria compared to the corresponding untreated control plants, at the three stages of growth $(75,90$, and 105 DAS). In most cases, the increments in growth parameters were often highly significant in comparison with untreated control plants. The most effective treatments on growth parameters were the interaction of FA at $9 \mathrm{gL}^{-1}$ with Sakha 4 cultivar followed by FA at $9 \mathrm{gL}^{-1}$ with Sakha 3 cultivar at the three stages of growth.

When considered as a single factor, foliar application of FA up to $9 \mathrm{gL}^{-1}$ increased $\mathrm{PH}, \mathrm{BN}, \mathrm{LN}$, TDW, 4-LA, LAI, SLW, and CGR compared to the untreated control plants.

Furthermore, the differences between cultivars were significant with respect to the above mentioned characters at the three physiological stages, except number of branches at 105 DAS, LAI at 75, and 105 DAS. Results cleared that "Sakha 4" cultivar was superior in the most growth characters followed by "Sakha 3" cultivar (Tables 1 and 2).

Regarding the interaction effect of FA and cultivars on vegetative growth, similar significant increases were obtained in the same growth parameters using different concentrations of FA and cultivars compared to control plants. The treatment FA at $9 \mathrm{gL}^{-1}$ with Sakha 4 cultivar enhanced the most growth parameters at the three stages of growth (Tables 1 and 2).

\section{Yield}

Data presented in Table 3 show that application of FA significantly increased faba bean yield and its components (i.e., PN/P, PW/P, SY/P, StY/P, SY/F, StY/F, and SI) more than untreated control plants at 115 DAS. The more promising results are obtained from $9 \mathrm{gL}^{-1}$ FA that were for seed yield (2.21 ton/fed) and straw yield (4.01 ton/fed).

Four faba bean cultivars, the increment in yield characters (i.e., PN/P, PW/P, SY/P, StY/P, SY/F, StY/F, and SI), were maximum with "Sakha 4" cultivar compared to other cultivars (Table 3). Furthermore, faba bean yield was far more sensitive to the interaction between FA and cultivars. The greatest increase in SY/F (i.e., increased 27.00 and $23.89 \%$ more than their controls, respectively) and StY/F (i.e., increased 38.97 and 33.23\%) was obtained by foliar application with $9 \mathrm{gL}^{-1} \mathrm{FA}$ and Sakha 4 cultivar, followed by $9 \mathrm{gL}^{-1} \mathrm{FA}$ and Sakha 3 cultivar for all measured parameters (Table 3). In all cases, the increments in faba bean yield were often significant in comparison with untreated controls (Table 3). 
Table 2 Effect of foliar spray application of fulvic acid on some growth characters of some faba bean varieties (combined analysis of two seasons)

\begin{tabular}{|c|c|c|c|c|c|c|c|c|c|c|c|c|}
\hline \multicolumn{13}{|c|}{ Growth characters of four faba bean cultivars } \\
\hline \multirow[t]{2}{*}{ Treatment } & \multicolumn{3}{|c|}{ Plant height $(\mathrm{cm})(\mathrm{PH})$} & \multicolumn{3}{|c|}{ No. branches plant $\left.{ }^{-1}\right)(\mathrm{BN})$} & \multicolumn{3}{|c|}{ No. leaves plant ${ }^{-1}(\mathrm{LN})$} & \multicolumn{3}{|c|}{ Total dry weight (TDW) (g plant ${ }^{-1}$ ) } \\
\hline & A & $B$ & C & A & $B$ & C & A & B & C & A & B & C \\
\hline \multicolumn{13}{|c|}{ Effect of fulvic acid } \\
\hline $0.0 \mathrm{gL}^{-1}$ & 59.31 & 72.61 & 90.78 & 3.64 & 4.50 & 5.67 & 45.71 & 52.40 & 59.33 & 20.92 & 39.60 & 49.80 \\
\hline $3.0 \mathrm{gL}^{-1}$ & 69.40 & 83.59 & 103.69 & 4.73 & 5.76 & 5.79 & 47.82 & 58.54 & 62.62 & 25.76 & 42.56 & 54.61 \\
\hline $6.0 \mathrm{gL}^{-1}$ & 78.35 & 96.68 & 110.50 & 4.52 & 4.70 & 5.87 & 50.90 & 62.63 & 65.10 & 29.67 & 48.50 & 59.96 \\
\hline $9.0 \mathrm{gL}^{-1}$ & 86.22 & 102.77 & 113.71 & 5.61 & 5.82 & 5.91 & 53.61 & 63.72 & 66.90 & 32.80 & 51.33 & 64.34 \\
\hline L. S.D. at 5\% & 5.01 & 7.39 & 3.91 & 1.01 & 1.12 & n.s. & 1.29 & 2.11 & n.s. & 2.96 & 3.12 & 3.81 \\
\hline \multicolumn{13}{|l|}{ Effect of cultivars } \\
\hline Nubaria 2 & 66.70 & 81.80 & 102.92 & 4.61 & 4.80 & 5.69 & 44.36 & 55.80 & 62.87 & 24.67 & 38.81 & 47.31 \\
\hline Sakha 1 & 60.91 & 71.96 & 89.83 & 3.62 & 3.78 & 4.83 & 42.45 & 51.39 & 60.68 & 21.11 & 34.70 & 44.57 \\
\hline Sakha 3 & 73.82 & 86.25 & 108.74 & 4.73 & 4.86 & 5.29 & 47.42 & 58.30 & 63.53 & 27.91 & 47.81 & 54.32 \\
\hline Sakha 4 & 76.73 & 91.34 & 112.65 & 4.84 & 4.95 & 5.59 & 49.39 & 60.51 & 64.42 & 29.18 & 49.51 & 59.39 \\
\hline L. S.D. at 5\% & 2.88 & 5.89 & 3.01 & 0.87 & 1.02 & n.s. & 5.80 & 1.89 & 0.90 & 2.58 & 3.01 & 2.44 \\
\hline \multicolumn{13}{|c|}{ Effect of interaction } \\
\hline \multicolumn{13}{|l|}{$0.0 \mathrm{gL}^{-1}$} \\
\hline Nubaria 2 & 68.66 & 80.46 & 109.56 & 4.72 & 4.84 & 5.50 & 45.12 & 55.20 & 59.70 & 22.39 & 41.60 & 52.30 \\
\hline Sakha 1 & 64.54 & 76.55 & 104.47 & 3.43 & 3.63 & 4.69 & 43.31 & 51.39 & 56.61 & 19.20 & 38.71 & 50.87 \\
\hline Sakha 3 & 71.45 & 84.64 & 113.38 & 4.76 & 4.82 & 5.85 & 46.05 & 57.43 & 60.52 & 27.91 & 46.81 & 55.32 \\
\hline Sakha 4 & 75.38 & 89.73 & 115.29 & 4.80 & 4.91 & 5.60 & 48.90 & 58.54 & 62.43 & 30.18 & 49.52 & 59.71 \\
\hline \multicolumn{13}{|l|}{$3.0 \mathrm{gL}^{-1}$} \\
\hline Nubaria 2 & 73.29 & 87.82 & 116.19 & 4.72 & 5.79 & 5.89 & 48.80 & 58.43 & 61.32 & 25.33 & 45.72 & 56.50 \\
\hline Sakha 1 & 66.61 & 81.91 & 109.40 & 3.61 & 4.81 & 5.49 & 45.74 & 55.52 & 59.20 & 21.10 & 40.83 & 53.47 \\
\hline Sakha 3 & 78.30 & 92.06 & 118.90 & 4.39 & 5.56 & 5.81 & 49.67 & 61.60 & 63.11 & 30.52 & 48.91 & 59.36 \\
\hline Sakha 4 & 82.95 & 89.42 & 122.81 & 4.94 & 5.66 & 5.92 & 52.58 & 64.71 & 66.93 & 33.61 & 51.80 & 63.74 \\
\hline \multicolumn{13}{|l|}{$6.0 \mathrm{gL}^{-1}$} \\
\hline Nubaria 2 & 79.87 & 92.21 & 118.72 & 5.52 & 6.60 & 7.39 & 50.40 & 59.72 & 62.83 & 28.39 & 47.66 & 59.96 \\
\hline Sakha 1 & 71.78 & 84.34 & 113.64 & 4.40 & 5.51 & 6.32 & 47.36 & 57.85 & 60.74 & 23.20 & 43.60 & 55.85 \\
\hline Sakha 3 & 80.67 & 96.79 & 120.56 & 5.67 & 6.59 & 7.49 & 57.27 & 62.85 & 64.60 & 31.43 & 50.07 & 64.18 \\
\hline Sakha 4 & 86.59 & 99.58 & 123.48 & 6.70 & 7.79 & 7.86 & 57.80 & 65.44 & 67.54 & 34.54 & 54.43 & 66.70 \\
\hline \multicolumn{13}{|l|}{$9.0 \mathrm{gL}^{-1}$} \\
\hline Nubaria 2 & 83.40 & 96.60 & 121.39 & 5.71 & 6.76 & 7.79 & 53.01 & 61.22 & 63.48 & 31.60 & 50.01 & 61.86 \\
\hline Sakha 1 & 77.39 & 89.76 & 116.20 & 4.69 & 5.59 & 6.66 & 49.92 & 59.33 & 61.34 & 26.48 & 47.80 & 59.70 \\
\hline Sakha 3 & 83.20 & 100.85 & 124.10 & 6.33 & 7.72 & 7.84 & 56.83 & 63.50 & 65.33 & 33.72 & 54.76 & 66.42 \\
\hline Sakha 4 & 86.61 & 106.94 & 125.33 & 7.16 & 7.89 & 7.93 & 59.74 & 66.61 & 68.43 & 37.29 & 58.32 & 68.39 \\
\hline L. S.D. at 5\% & 2.93 & 3.89 & 2.06 & 1.02 & n.s. & n.s. & 2.16 & 1.87 & n.s. & 2.90 & 1.97 & 2.02 \\
\hline
\end{tabular}

$A$ after 75 days from sowing, $B$ after 90 days from sowing, $C$ after 105 days from sowing

\section{Photosynthetic pigments}

Data presented in Table 4 show that Chl $a$ and $b$, carotenoids, and TPP in the leaves of four faba bean cultivars reached a maximum value at 90 DAS. Generally, foliar application of FA at any concentration significantly increased the Chl $a$ and $b$, carotenoids, and consequently the TPP more than controls at 75, 90, and 105 DAS.
The most effective concentration was $9 \mathrm{gL}^{-1}$ of FA, Sakha 4 cultivar, and their interaction at the three stages of growth (Table 4).

Foliar spray of faba bean plants at all three FA concentrations $\left(3,6\right.$, and $\left.9 \mathrm{gL}^{-1}\right)$ tested significantly increased TPP more than untreated control plants (Table 4). The highest recorded value of Chl $a$ and $b$, carotenoids, and 
Table 3 Effect of foliar spray application of fulvic acid on some growth characters of some faba bean varieties (combined analysis of two seasons)

\begin{tabular}{|c|c|c|c|c|c|c|c|c|c|c|}
\hline \multicolumn{11}{|c|}{ Growth characters of four faba bean cultivars } \\
\hline \multirow[t]{2}{*}{ Treatment } & \multicolumn{3}{|c|}{ Fourth leaf area $\left(\mathrm{cm}^{2}\right)(\mathrm{LA})$} & \multicolumn{3}{|c|}{ Leaf area index (LAI) } & \multicolumn{2}{|c|}{ Specific leaf Weight (SLW) $\left(\mathrm{mg} / \mathrm{cm}^{2} /\right.$ day) } & \multicolumn{2}{|c|}{ Crop growth rate $(\mathrm{CGR})\left(\mathrm{mg} / \mathrm{cm}^{2} /\right.$ day) } \\
\hline & A & B & C & A & $\mathrm{B}$ & C & $A-B$ & $\mathrm{~B}-\mathrm{C}$ & $A-B$ & $B-C$ \\
\hline \multicolumn{11}{|c|}{ Effect of fulvic acid } \\
\hline $0.0 \mathrm{gL}^{-1}$ & 1144.94 & 1391.56 & 1589.78 & 1.86 & 2.01 & 2.92 & 3.08 & 3.33 & 1411.26 & 1359.57 \\
\hline $3.0 \mathrm{gL}^{-1}$ & 1241.90 & 1501.33 & 1770.13 & 1.96 & 2.54 & 3.11 & 3.81 & 4.76 & 1579.57 & 1452.92 \\
\hline $6.0 \mathrm{gL}^{-1}$ & 1380.58 & 1621.01 & 1867.64 & 1.92 & 2.91 & 3.31 & 4.70 & 5.46 & 1669.46 & 1467.80 \\
\hline $9.0 \mathrm{gL}^{-1}$ & 1497.01 & 1722.31 & 1998.46 & 1.99 & 3.15 & 3.36 & 5.29 & 5.72 & 1770.39 & 1586.26 \\
\hline L. S.D. at $5 \%$ & 76.06 & 87.33 & 96.51 & n.s. & 0.26 & 0.13 & 0.53 & 1.01 & 78.66 & 92.43 \\
\hline \multicolumn{11}{|l|}{ Effect of cultivars } \\
\hline Nubaria 2 & 1251.80 & 1452.42 & 1553.33 & 1.48 & 2.54 & 3.01 & 4.72 & 4.29 & 1401.47 & 1330.21 \\
\hline Sakha 1 & 1143.66 & 1390.71 & 1476.18 & 1.41 & 2.01 & 2.89 & 3.67 & 3.31 & 1381.58 & 1302.03 \\
\hline Sakha 3 & 1296.49 & 1532.01 & 1649.81 & 1.55 & 2.82 & 3.07 & 4.69 & 5.56 & 1467.60 & 1344.92 \\
\hline Sakha 4 & 1371.83 & 1650.31 & 1755.76 & 1.67 & 3.01 & 3.12 & 4.89 & 5.80 & 1572.70 & 1439.81 \\
\hline L. S.D. at $5 \%$ & 57.34 & 71.13 & 82.60 & n.s. & 0.18 & n.s. & 0.44 & 0.59 & 66.30 & 80.51 \\
\hline \multicolumn{11}{|c|}{ Effect of interaction } \\
\hline \multicolumn{11}{|l|}{$0.0 \mathrm{gL}^{-1}$} \\
\hline Nubaria 2 & 1150.78 & 1552.40 & 1778.66 & 1.97 & 2.56 & 3.10 & 3.67 & 4.44 & 1390.47 & 1336.14 \\
\hline Sakha 1 & 1131.77 & 1468.39 & 1690.82 & 1.92 & 2.40 & 3.07 & 3.52 & 3.21 & 1286.40 & 1251.39 \\
\hline Sakha 3 & 1196.51 & 1662.73 & 1887.44 & 1.98 & 3.15 & 3.20 & 4.09 & 4.60 & 1470.51 & 1366.51 \\
\hline Sakha 4 & 1249.80 & 1679.82 & 1909.30 & 1.99 & 3.22 & 3.29 & 4.59 & 5.04 & 1544.30 & 1440.36 \\
\hline \multicolumn{11}{|l|}{$3.0 \mathrm{gL}^{-1}$} \\
\hline Nubaria 2 & 1152.29 & 1650.68 & 1870.39 & 1.99 & 3.17 & 3.26 & 4.59 & 4.53 & 1467.35 & 1359.50 \\
\hline Sakha 1 & 1139.66 & 1532.70 & 1834.60 & 1.97 & 2.69 & 3.03 & 3.96 & 3.84 & 1390.46 & 1256.83 \\
\hline Sakha 3 & 1260.38 & 1716.59 & 1980.27 & 2.07 & 3.39 & 3.41 & 4.69 & 5.12 & 1587.31 & 1396.53 \\
\hline Sakha 4 & 1371.49 & 1733.40 & 1996.36 & 2.11 & 3.52 & 3.59 & 5.72 & 5.49 & 1697.49 & 1477.40 \\
\hline \multicolumn{11}{|l|}{$6.0 \mathrm{gL}^{-1}$} \\
\hline Nubaria 2 & 1290.58 & 1741.26 & 1957.64 & 2.01 & 3.26 & 3.29 & 4.71 & 4.58 & 1579.57 & 1353.92 \\
\hline Sakha 1 & 1181.63 & 1636.19 & 1866.82 & 1.99 & 2.94 & 3.15 & 4.54 & 3.89 & 1456.68 & 1340.01 \\
\hline Sakha 3 & 1310.49 & 1759.35 & 2011.72 & 2.09 & 3.41 & 3.50 & 5.66 & 5.60 & 1669.46 & 1467.83 \\
\hline Sakha 4 & 1411.30 & 1869.43 & 2126.28 & 2.11 & 3.56 & 3.66 & 5.76 & 5.69 & 1776.35 & 1587.74 \\
\hline \multicolumn{11}{|l|}{$9.0 \mathrm{gL}^{-1}$} \\
\hline Nubaria 2 & 1352.74 & 1841.36 & 1998.21 & 2.06 & 3.33 & 3.38 & 4.79 & 4.62 & 1689.47 & 1470.51 \\
\hline Sakha 1 & 1259.82 & 1770.47 & 1990.49 & 2.01 & 3.19 & 3.27 & 4.59 & 3.93 & 1588.56 & 1441.60 \\
\hline Sakha 3 & 1366.62 & 1870.29 & 2055.63 & 2.09 & 3.44 & 3.54 & 5.81 & 4.98 & 1779.38 & 1582.48 \\
\hline Sakha 4 & 1533.52 & 2029.01 & 2248.54 & 2.11 & 3.59 & 3.68 & 5.92 & 5.81 & 1889.29 & 1593.33 \\
\hline L. S.D. at 5\% & 67.90 & 89.34 & 92.30 & n.s. & 0.12 & n.s. & 0.24 & 0.62 & 84.36 & 96.78 \\
\hline
\end{tabular}

$A$ after 75 days from sowing, $B$ after 90 days from sowing, $C$ after 105 days from sowing

TPP in the leaves of faba bean was obtained with $9 \mathrm{gL}^{-1}$ at the three stages of growth, also Sakha 4 cultivar recorded the highest values of these parameters.

Moreover, the interaction between FA and cultivars increased Chl $a$ and $b$, carotenoids, and TPP in the leaves more than control plants and there were significant effects between treatments at all three stages of growth except
Chl $a$ at 90 DAS and carotenoids at 105 DAS. Data also show the most effective treatment was FA at $9 \mathrm{gL}^{-1}$ when used with Sakha 4 cultivar (Table 4).

\section{Chemical constituents}

Data presented in Table 5 show that, foliar application of FA at any concentration and their combination significantly 
Table 4 Effect of foliar spray application of fulvic acid on some growth characters of some faba bean varieties (combined analysis of two seasons)

\begin{tabular}{|c|c|c|c|c|c|c|c|c|c|}
\hline \multicolumn{10}{|c|}{ Yield and yield components of four faba bean cultivars } \\
\hline \multirow[t]{2}{*}{ Treatment } & \multirow{2}{*}{$\begin{array}{l}\text { Plant height } \\
(\mathrm{cm})(\mathrm{PH})\end{array}$} & \multicolumn{2}{|l|}{ No. plant $^{-1}$} & \multirow{2}{*}{$\begin{array}{l}\text { Weight of pods } \\
\left(\text { g plant }^{-1}\right)(\text { PW) }\end{array}$} & \multicolumn{2}{|c|}{ Yield (g plant ${ }^{-1}$ ) } & \multicolumn{2}{|c|}{ Yield/fed. (ton) } & \multirow{2}{*}{$\begin{array}{l}\text { Seed Index } \\
\text { (g) (SI) }\end{array}$} \\
\hline & & Branches (BN) & Pods (PN) & & Seed (SY/P) & $\overline{\text { Straw }(\mathrm{StY} / \mathrm{P})}$ & Seed (SY/F) & Straw (StY/F) & \\
\hline \multicolumn{10}{|c|}{ Effect of fulvic acid } \\
\hline $0.0 \mathrm{gL}^{-1}$ & 118.51 & 4.96 & 29.91 & 80.71 & 50.90 & 86.59 & 1.84 & 3.20 & 69.90 \\
\hline $3.0 \mathrm{gL}^{-1}$ & 121.90 & 5.23 & 31.44 & 86.60 & 54.31 & 98.77 & 1.93 & 3.56 & 75.43 \\
\hline $6.0 \mathrm{gL}^{-1}$ & 124.82 & 6.12 & 33.36 & 89.96 & 58.56 & 109.92 & 2.06 & 3.86 & 84.92 \\
\hline $9.0 \mathrm{gL}^{-1}$ & 126.76 & 7.01 & 35.21 & 94.85 & 68.27 & 115.81 & 2.21 & 4.01 & 89.50 \\
\hline L. S.D. at $5 \%$ & 3.29 & 0.61 & 2.30 & 4.36 & 3.41 & 8.82 & 0.16 & 0.21 & 4.53 \\
\hline \multicolumn{10}{|l|}{ Effect of cultivars } \\
\hline Nubaria 2 & 120.67 & 5.60 & 28.96 & 81.27 & 53.31 & 107.09 & 1.87 & 3.42 & 76.60 \\
\hline Sakha 1 & 116.55 & 4.81 & 26.47 & 76.50 & 50.64 & 99.42 & 1.76 & 3.21 & 72.86 \\
\hline Sakha 3 & 122.40 & 5.72 & 31.39 & 87.46 & 56.51 & 110.79 & 1.92 & 3.78 & 80.76 \\
\hline Sakha 4 & 123.26 & 6.33 & 33.66 & 90.50 & 57.80 & 112.29 & 2.01 & 3.96 & 86.40 \\
\hline L.S.D. at $5 \%$ & 2.92 & 0.42 & 1.29 & 3.04 & 2.96 & 2.96 & 0.11 & 0.18 & 3.48 \\
\hline \multicolumn{10}{|c|}{ Effect of interaction } \\
\hline \multicolumn{10}{|l|}{$0.0 \mathrm{gL}^{-1}$} \\
\hline Nubaria 2 & 117.89 & 4.98 & 27.98 & 78.90 & 51.42 & 100.21 & 1.69 & 3.01 & 75.01 \\
\hline Sakha 1 & 115.90 & 4.44 & 25.92 & 70.56 & 49.64 & 97.56 & 1.62 & 2.96 & 71.80 \\
\hline Sakha 3 & 119.85 & 5.03 & 32.12 & 90.71 & 54.23 & 109.87 & 1.80 & 3.22 & 78.69 \\
\hline Sakha 4 & 120.77 & 5.12 & 32.76 & 92.12 & 56.67 & 113.29 & 2.00 & 3.31 & 82.56 \\
\hline \multicolumn{10}{|l|}{$3.0 \mathrm{gL}^{-1}$} \\
\hline Nubaria 2 & 120.60 & 5.67 & 29.40 & 83.60 & 54.70 & 108.90 & 1.78 & 3.15 & 78.23 \\
\hline Sakha 1 & 116.39 & 4.87 & 27.55 & 77.39 & 50.57 & 99.38 & 1.68 & 3.06 & 73.41 \\
\hline Sakha 3 & 120.71 & 5.64 & 32.90 & 94.10 & 56.38 & 111.33 & 1.89 & 3.49 & 83.05 \\
\hline Sakha 4 & 122.82 & 6.02 & 33.64 & 96.47 & 59.29 & 116.47 & 2.12 & 3.59 & 86.92 \\
\hline \multicolumn{10}{|l|}{$6.0 \mathrm{gL}^{-1}$} \\
\hline Nubaria 2 & 121.33 & 5.85 & 32.60 & 91.70 & 56.69 & 112.80 & 1.89 & 3.53 & 82.68 \\
\hline Sakha 1 & 118.46 & 5.32 & 28.86 & 81.46 & 52.12 & 103.12 & 1.75 & 3.20 & 78.37 \\
\hline Sakha 3 & 122.55 & 6.15 & 34.80 & 98.01 & 58.80 & 115.41 & 2.09 & 3.88 & 87.66 \\
\hline Sakha 4 & 124.92 & 7.21 & 35.71 & 99.43 & 62.72 & 120.72 & 2.21 & 4.11 & 89.50 \\
\hline \multicolumn{10}{|l|}{$9.0 \mathrm{gL}^{-1}$} \\
\hline Nubaria 2 & 122.89 & 6.34 & 33.87 & 96.90 & 59.55 & 116.93 & 1.96 & 3.89 & 85.44 \\
\hline Sakha 1 & 120.60 & 5.86 & 30.52 & 86.67 & 55.60 & 107.56 & 1.92 & 3.78 & 79.30 \\
\hline Sakha 3 & 124.41 & 6.49 & 35.90 & 99.80 & 65.46 & 122.46 & 2.23 & 4.29 & 88.29 \\
\hline Sakha 4 & 126.22 & 7.46 & 37.41 & 103.60 & 69.27 & 129.27 & 2.54 & 4.60 & 91.56 \\
\hline L.S.D. at $5 \%$ & 3.55 & 0.77 & 2.68 & 5.01 & 3.95 & 5.74 & 0.20 & 0.34 & 4.67 \\
\hline
\end{tabular}

increased the total carbohydrates percentage, crude protein percentage, minerals (nitrogen, phosphorus, and potassium) percentage, arginine, lysine, phenylalanine, tryptophan, and consequently total detected free amino acids $\left(\mathrm{gkg}^{-1} \mathrm{DW}\right)$ in the dry seeds of faba bean cultivars compared with their control at 115 DAS (i.e., the harvest date). The most effective treatment was FA at $9 \mathrm{gL}^{-1}$, Sakha 4 cultivar, or their interaction.
Furthermore, the results obtained indicate that the highest level of total carbohydrates (59.71, 55.47, and $60.29 \%$ ), crude protein $(33.51,31.27$, and $35.09 \%)$, total detected free amino acids $(17.93,16.33$, and $20.20 \mathrm{~g} / \mathrm{Kg}$ DW), nitrogen $(5.39,4.87$, and $5.62 \%)$, phosphorus $(0.59$, 0.46 , and $0.57 \%)$, and potassium $(0.94,0.87$, and $0.96 \%$ ) was obtained for either $9 \mathrm{gL}^{-1} \mathrm{FA}$, Sakha 4 seeds, or their interaction, respectively compared with $49.87 \%$, $25.90 \%$, 
Table 5 Effect of foliar spray application of fulvic acid on some growth characters of some faba bean varieties (combined analysis of two seasons)

\begin{tabular}{|c|c|c|c|c|c|c|c|c|c|c|c|c|}
\hline \multirow[t]{2}{*}{ Treatment } & \multicolumn{4}{|c|}{75 days after sowing } & \multicolumn{4}{|c|}{90 days after sowing } & \multicolumn{4}{|c|}{105 days } \\
\hline & Chl. a & Chl. b & Carotenoids & $\begin{array}{l}\text { Total } \\
\text { pigments }\end{array}$ & Chl. a & Chl. b & Carotenoids & $\begin{array}{l}\text { Total } \\
\text { pigments }\end{array}$ & $\overline{C h l . a}$ & Chl. b & Carotenoids & $\begin{array}{l}\text { Total } \\
\text { pigments }\end{array}$ \\
\hline \multicolumn{13}{|c|}{ Effect of fulvic acid } \\
\hline $0.0 \mathrm{gL}^{-1}$ & 1.64 & 0.54 & 0.50 & 2.68 & 2.01 & 1.18 & 1.32 & 4.51 & 1.96 & 0.92 & 1.01 & 3.89 \\
\hline $3.0 \mathrm{gL}^{-1}$ & 1.74 & 0.66 & 0.64 & 3.04 & 2.09 & 1.21 & 1.36 & 4.66 & 2.05 & 1.13 & 1.09 & 4.27 \\
\hline $6.0 \mathrm{gL}^{-1}$ & 1.84 & 0.72 & 0.76 & 3.39 & 2.33 & 1.30 & 1.41 & 5.04 & 2.07 & 1.19 & 1.06 & 4.32 \\
\hline $9.0 \mathrm{gL}^{-1}$ & 1.87 & 1.08 & 1.13 & 4.08 & 2.51 & 1.33 & 1.47 & 5.31 & 2.22 & 1.29 & 1.14 & 4.65 \\
\hline L.S.D. at $5 \%$ & 0.10 & 0.12 & 0.15 & 0.33 & 0.18 & 0.09 & 0.06 & 0.27 & 0.08 & 0.10 & 0.13 & 0.15 \\
\hline \multicolumn{13}{|l|}{ Effect of cultivars } \\
\hline Nubaria 2 & 1.69 & 0.61 & 0.59 & 2.89 & 2.05 & 0.92 & 1.12 & 4.09 & 1.99 & 0.91 & 1.02 & 3.92 \\
\hline Sakha 1 & 1.62 & 0.55 & 0.52 & 2.69 & 1.97 & 0.81 & 1.08 & 3.86 & 2.01 & 0.98 & 1.06 & 4.05 \\
\hline Sakha 3 & 1.77 & 0.69 & 0.63 & 3.09 & 2.31 & 1.21 & 1.39 & 4.91 & 2.11 & 1.09 & 1.08 & 4.28 \\
\hline Sakha 4 & 1.83 & 1.11 & 1.08 & 4.02 & 2.40 & 1.36 & 1.44 & 5.20 & 2.18 & 1.26 & 1.10 & 4.54 \\
\hline L.S.D. at $5 \%$ & 0.06 & 0.11 & 0.14 & 0.21 & 0.08 & 0.11 & 0.13 & 0.29 & 0.11 & 0.16 & n.s. & 0.27 \\
\hline \multicolumn{13}{|c|}{ Effect of interaction } \\
\hline \multicolumn{13}{|l|}{$0.0 \mathrm{gL}^{-1}$} \\
\hline Nubaria 2 & 1.53 & 0.40 & 0.43 & 2.36 & 1.96 & 0.90 & 1.02 & 3.91 & 2.02 & 1.02 & 1.04 & 4.08 \\
\hline Sakha 1 & 1.60 & 0.54 & 0.50 & 2.64 & 2.01 & 1.09 & 1.05 & 4.15 & 2.00 & 1.00 & 1.01 & 4.01 \\
\hline Sakha 3 & 1.71 & 0.64 & 0.61 & 2.96 & 2.12 & 1.20 & 1.10 & 4.42 & 2.09 & 1.13 & 1.07 & 4.29 \\
\hline Sakha 4 & 1.80 & 1.07 & 1.05 & 3.92 & 2.24 & 1.29 & 1.19 & 4.72 & 2.11 & 1.15 & 1.12 & 4.38 \\
\hline \multicolumn{13}{|l|}{$3.0 \mathrm{gL}^{-1}$} \\
\hline Nubaria 2 & 1.76 & 0.69 & 0.64 & 3.09 & 2.11 & 1.12 & 1.08 & 4.31 & 2.11 & 1.11 & 1.09 & 4.31 \\
\hline Sakha 1 & 1.66 & 0.59 & 0.56 & 2.81 & 2.09 & 1.06 & 1.01 & 4.16 & 2.09 & 1.06 & 1.01 & 4.16 \\
\hline Sakha 3 & 1.81 & 0.72 & 0.69 & 3.22 & 2.30 & 1.19 & 1.22 & 4.71 & 2.18 & 1.15 & 1.10 & 4.43 \\
\hline Sakha 4 & 1.90 & 1.21 & 1.12 & 4.23 & 2.41 & 1.28 & 1.26 & 4.95 & 2.22 & 1.17 & 1.12 & 4.51 \\
\hline \multicolumn{13}{|l|}{$6.0 \mathrm{gL}^{-1}$} \\
\hline Nubaria 2 & 1.84 & 0.72 & 0.76 & 3.32 & 2.40 & 1.21 & 1.19 & 4.80 & 2.13 & 1.19 & 1.16 & 4.48 \\
\hline Sakha 1 & 1.78 & 0.69 & 0.63 & 3.10 & 2.22 & 1.18 & 1.12 & 4.52 & 2.11 & 1.15 & 1.03 & 4.29 \\
\hline Sakha 3 & 2.01 & 1.23 & 1.20 & 4.44 & 2.46 & 1.70 & 1.25 & 5.41 & 2.20 & 1.18 & 1.12 & 4.50 \\
\hline Sakha 4 & 2.09 & 1.29 & 1.26 & 4.64 & 2.51 & 2.01 & 1.34 & 5.86 & 2.30 & 1.26 & 1.20 & 4.76 \\
\hline \multicolumn{13}{|l|}{$9.0 \mathrm{gL}^{-1}$} \\
\hline Nubaria 2 & 1.86 & 1.06 & 1.11 & 4.03 & 2.41 & 1.56 & 1.09 & 5.06 & 2.33 & 1.29 & 1.09 & 4.71 \\
\hline Sakha 1 & 1.79 & 0.73 & 0.70 & 3.22 & 2.31 & 1.29 & 1.03 & 4.63 & 2.08 & 1.16 & 1.02 & 4.26 \\
\hline Sakha 3 & 2.11 & 1.31 & 1.23 & 4.65 & 2.50 & 2.05 & 1.39 & 5.94 & 2.38 & 1.56 & 1.08 & 5.02 \\
\hline Sakha 4 & 2.20 & 1.33 & 1.30 & 4.83 & 2.57 & 2.19 & 1.78 & 6.54 & 2.41 & 1.59 & 1.19 & 5.19 \\
\hline L.S.D. at $5 \%$ & 0.09 & 0.18 & 0.31 & 0.43 & n.s. & 0.12 & 0.27 & 0.30 & 0.13 & 0.18 & n.s. & 0.28 \\
\hline
\end{tabular}

$13.52 \mathrm{~g} / \mathrm{Kg} \mathrm{DW}, 4.58 \%$, $0.39 \%$, and $0.62 \%$ for controls (Table 5).

\section{Discussion}

The present study indicates that the application of FA up to $9 \mathrm{gL}^{-1}$ and four faba bean (Noubaria 2, Sakha 1, Sakha 3, and Sakha 4) cultivars, individually or in combination, greatly promoted the vegetative growth and dry matter production at the three stages of growth by increasing the development of shoots and roots, increasing leaf area and Chl. accumulation. Promotion of root growth enhance capacity to uptake and utilize nutrients from the soil determining higher dry matter accumulation. In this connection, fulvic acid enhanced the numbers of root initials on hypocotyl sections of common bean, increased root elongation of maize (Eyheraguibel et al. 
2008), number and length of lateral roots of Arabidopis, and micro-Tom tomato (Dobbss et al. 2007). FAs play roles as natural chelators in the mobilization, and transport of $\mathrm{Fe}$ and other micronutrients (Bocanegra et al. 2006) increased chlorophyll content in soybean and ryegrass (Chen et al. 2004), photosynthesis rate in maize (Anjum et al. 2011), interacted with the signaling pathway for plant hormones, and increased intercellular levels of ATP and glucose-6-phosphate that were related to growth promotion in cell cultures of Greek fir (Zancani et al. 2011). However, the application of FA has only been reported in a few cases: maize (Eyheraguibel et al. 2008) and wheat (Anjum et al. 2011). Intuitively, the combination of FA and cultivars more effectively increased growth and dry matter production of faba bean than single treatments.

Faba bean yield and its components (i.e., PN/P, PW/P, SY/P, StY/P, SY/F, StY/F, and SI) and quality of seeds was significantly enhanced by FA, especially at $9 \mathrm{gL}^{-1}$. FA via enhanced photosynthetic activity, increased vegetative growth, dry matter accumulation, and consequently increased translocation and accumulation of certain metabolites in plant organs, which affected their yield and yield components (Table 3). In this respect, soil applications of FA enhanced flower number of cucumber plant (Rauthan and Schnitzer 1981), fruit weight, and quality of lemon trees (Citrus limon) (Sánchez-Sánchez et al. 2002) and pepper (Aminifard et al. 2012) and increased yield of maize (Anjum et al. 2011). The interaction between FA and cultivars was more effective in improving yield and quality of pods; FA at $9 \mathrm{gL}^{-1}$ with Sakha 4 cultivar increased yield and quality of pods more effectively than other cultivars (Table 3 ).

Photosynthetic pigments in faba bean leaves were significantly enhanced by the application of all FA and/or their interaction at 75, 90, and 105 DAS (Table 4). Fulvic acid might increase the uptake of $\mathrm{N}$ and $\mathrm{Mg}$ (structural component of $\mathrm{Chl}$ ), retarded senescence, and enhanced $\mathrm{Chl}$ accumulation, which would lead to a greater rate of photosynthesis. In accordance, humic acid and phosphorous elevated $\mathrm{N}, \mathrm{Fe}$, and $\mathrm{Mg}$ ion contents in bell pepper (Capsicum annuum L. cv. Demre) shoots under mild salinity stress (Çimrin et al. 2010). The promotive effects of FAs on chlorophyll content and net photosynthesis rates were observed in soybean and ryegrass (Chen et al. 2004) and in maize (Anjum et al. 2011). However, Sakha 4 cultivar recorded the maximum value of $C h l a$ and $b$, carotenoids, and TPP in the leaves compared with other cultivars. Therefore, the combination of FA at $9 \mathrm{gL}^{-1}$ and Sakha 4 cultivar was the best treatment to increase the TPP of leaves at the three stages of growth.

A foliar application of FA up to $9 \mathrm{gL}^{-1}$, four faba bean cultivars "Noubaria 2, Sakha 1, Sakha 3, and Sakha 4" or their interactions significantly increased the CP, CPP, FAA (Arg; Lys, Phe, and Try), N, P, and K content and consequently enhanced seeds quality at the harvest date, possibly due to the bioregulatory effect of FA on enzymatic activity and translocation processes from leaves to seeds, linking, or converting to other plant metabolites. Similarly, FA enhanced pepper fruit quality, including total soluble solids, antioxidant activity, total phenolic, carbohydrates, capsaicin, and carotenoids (Aminifard et al. 2012). Biostimulants improve the primary metabolism of plants, increasing the levels of carbohydrates, proteins biosynthesis, free amino acids, pigments, and various enzymes (Yakhin et al. 2017). However, Sakha 4 cultivar was more effective than other cultivars in increasing the level of total carbohydrates, crude protein, total free amino acids, nitrogen, phosphorus, and potassium content in the dry seeds (Table 5).

Thus, the treatment FA at $9 \mathrm{gL}^{-1}$ with Sakha 4 cultivar, followed by FA at $9 \mathrm{gL}^{-1}$ with Sakha 3 cultivar, were very effective for enhancing the level of lysine, phenylalanine, and tryptophan (essential amino acids) and arginine (non-essential amino acids) (Table 5). Enriching crop plants with essential amino acids (particularly Lys., Trp., and Met.) has both economical and humanitarian interest due to inability of humans and many farm animals to synthesize these essential amino acids (Ufaz and Galili 2008). Humic substances enhanced the expression of the phenylalanine (tyrosine) ammonia-lyase (PAL/TAL) that catalyzes the first main step in the biosynthesis of phenolic and accumulation in leaves by converting phenylalanine to trans-cinnamic acid and tyrosine to $p$-coumaric acid (Schiavon et al. 2010) in tomato leaves (Olivares et al. 2015). Phenolic compounds serve diverse functions in plants, including protection against biotic and abiotic stresses, cellular signaling, and mechanical support (MacDonald and D'Cunha 2007). Tryptophan is a precursor of indole-3-acetic acid (IAA) (Radwanski and Last 1995). IAA plays a critical role in plant growth and development by influencing many plant functions and expression of specific plant genes (Calvo et al. 2014). Ornithine and arginine can be used in higher plants for biosynthesis of the polyamine putresine through ornithine decarboxylation and putresine may be critical in regulation of development processes (Walden et al. 1997). Polyamines may act to store organic nitrogen (Flore and Filner 1985) and play a role in the regulation of DNA replication, cell division, and implicated in the control of senescence and morphogenesis (Galston and Sawhney 1990). In our study, treatment FA at $9 \mathrm{gL}^{-1}$ with Sakha 4 cultivar significantly increased N, P, and $\mathrm{K}$ content in the dry seeds. Similarly, Fulvic acids enhanced the uptake of $\mathrm{N}, \mathrm{P}, \mathrm{K}, \mathrm{Ca}, \mathrm{Mg}, \mathrm{Cu}, \mathrm{Fe}$, and $\mathrm{Zn}$ in cucumber plants (Rauthan and Schnitzer (1981), phosphate in beech (Fagus sylvatica) (Asp and Berggren 1990), increased $\mathrm{N}$ content in wild olive (Olea europaea) and maize plants (Murillo et al. 2005; Eyheraguibel et al. 2008) and play roles as natural chelators in the mobilization and transport of Fe and other micronutrients (Bocanegra et al. 2006). Thus, the yield-contributing characters and yield of 
Table 6 Effect of foliar spray application of fulvic acid on some growth characters of some faba bean varieties (combined analysis of two seasons)

\begin{tabular}{|c|c|c|c|c|c|c|c|c|c|c|}
\hline \multirow[t]{3}{*}{ Treatment } & \multicolumn{10}{|c|}{ Seeds' chemical constituent of four faba bean cultivars } \\
\hline & \multirow{2}{*}{$\begin{array}{l}\text { Total } \\
\text { carbohydrate \% }\end{array}$} & \multirow{2}{*}{$\begin{array}{l}\text { Crude } \\
\text { protein \% }\end{array}$} & \multicolumn{5}{|c|}{ Amino acids $\mathrm{g} \mathrm{kg}^{-1} \mathrm{DW}$} & \multirow[t]{2}{*}{$\mathrm{N} \%$} & \multirow[t]{2}{*}{$\mathrm{P} \%$} & \multirow[t]{2}{*}{$K \%$} \\
\hline & & & Arginine & Lysine & Phenylalanine & Tryptophan & Total detected amino acids & & & \\
\hline \multicolumn{11}{|c|}{ Effect of fulvic acid } \\
\hline $0.0 \mathrm{gL}^{-1}$ & 49.87 & 25.90 & 5.93 & 2.81 & 3.69 & 1.09 & 13.52 & 4.58 & 0.39 & 0.62 \\
\hline $3.0 \mathrm{gL}^{-1}$ & 52.41 & 28.88 & 6.81 & 3.01 & 3.98 & 1.39 & 15.19 & 4.69 & 0.41 & 0.78 \\
\hline $6.0 \mathrm{gL}^{-1}$ & 56.39 & 31.80 & 7.19 & 3.47 & 4.39 & 1.46 & 16.51 & 4.89 & 0.48 & 0.83 \\
\hline $9.0 \mathrm{gL}^{-1}$ & 59.71 & 33.51 & 7.90 & 3.69 & 4.85 & 1.49 & 17.93 & 5.39 & 0.59 & 0.94 \\
\hline L. S.D. at $5 \%$ & 1.71 & 1.89 & 0.36 & 0.21 & 0.23 & 0.02 & 1.18 & 0.11 & 0.02 & 0.05 \\
\hline \multicolumn{11}{|l|}{ Effect of cultivars } \\
\hline Nubaria 2 & 51.90 & 26.09 & 6.39 & 3.01 & 4.32 & 1.14 & 14.86 & 4.49 & 0.38 & 0.64 \\
\hline Sakha 1 & 50.77 & 25.39 & 5.90 & 2.89 & 3.12 & 1.01 & 12.92 & 3.62 & 0.35 & 0.59 \\
\hline Sakha 3 & 53.38 & 29.46 & 6.88 & 3.12 & 4.36 & 1.18 & 15.54 & 4.59 & 0.42 & 0.72 \\
\hline Sakha 4 & 55.47 & 31.27 & 7.02 & 3.34 & 4.76 & 1.21 & 16.33 & 4.87 & 0.46 & 0.87 \\
\hline L. S.D. at $5 \%$ & 1.13 & 1.21 & 0.14 & 0.23 & 0.18 & n.s. & 0.82 & 0.19 & 0.03 & 0.04 \\
\hline \multicolumn{11}{|c|}{ Effect of interaction } \\
\hline \multicolumn{11}{|l|}{$0.0 \mathrm{gL}^{-1}$} \\
\hline Nubaria 2 & 50.46 & 27.90 & 6.78 & 3.30 & 4.10 & 1.23 & 15.41 & 4.12 & 0.35 & 0.57 \\
\hline Sakha 1 & 49.38 & 26.84 & 5.41 & 2.92 & 3.67 & 1.12 & 13.12 & 3.82 & 0.31 & 0.53 \\
\hline Sakha 3 & 52.66 & 29.50 & 6.30 & 3.44 & 4.59 & 1.30 & 15.63 & 4.76 & 0.39 & 0.62 \\
\hline Sakha 4 & 54.71 & 31.71 & 6.72 & 3.87 & 4.87 & 1.34 & 16.80 & 4.87 & 0.42 & 0.67 \\
\hline \multicolumn{11}{|l|}{$3.0 \mathrm{gL}^{-1}$} \\
\hline Nubaria 2 & 53.90 & 30.51 & 6.98 & 3.55 & 4.29 & 1.30 & 16.12 & 4.54 & 0.40 & 0.62 \\
\hline Sakha 1 & 51.72 & 27.36 & 5.93 & 3.12 & 4.22 & 1.18 & 14.45 & 4.29 & 0.36 & 0.56 \\
\hline Sakha 3 & 54.47 & 31.44 & 6.87 & 3.67 & 4.68 & 1.34 & 16.56 & 4.92 & 0.41 & 0.69 \\
\hline Sakha 4 & 57.38 & 33.27 & 7.44 & 4.01 & 5.21 & 1.39 & 18.05 & 5.00 & 0.45 & 0.71 \\
\hline \multicolumn{11}{|l|}{$6.0 \mathrm{gL}^{-1}$} \\
\hline Nubaria 2 & 55.62 & 31.76 & 7.50 & 3.77 & 4.43 & 1.34 & 17.04 & 4.69 & 0.43 & 0.69 \\
\hline Sakha 1 & 53.45 & 29.47 & 6.98 & 3.39 & 4.28 & 1.25 & 15.90 & 4.44 & 0.38 & 0.58 \\
\hline Sakha 3 & 57.40 & 32.36 & 7.89 & 4.15 & 5.33 & 1.40 & 18.77 & 5.23 & 0.46 & 0.78 \\
\hline Sakha 4 & 59.63 & 34.40 & 8.34 & 4.51 & 5.50 & 1.46 & 19.81 & 5.49 & 0.50 & 0.81 \\
\hline \multicolumn{11}{|l|}{$9.0 \mathrm{gL}^{-1}$} \\
\hline Nubaria 2 & 57.62 & 32.50 & 7.59 & 4.01 & 4.55 & 1.37 & 17.52 & 5.04 & 0.47 & 0.74 \\
\hline Sakha 1 & 54.80 & 30.64 & 7.01 & 3.96 & 4.39 & 1.29 & 16.65 & 4.81 & 0.42 & 0.64 \\
\hline Sakha 3 & 59.43 & 33.11 & 7.92 & 4.76 & 5.56 & 1.44 & 19.68 & 5.35 & 0.53 & 0.82 \\
\hline Sakha 4 & 60.29 & 35.09 & 8.11 & 4.90 & 5.71 & 1.48 & 20.20 & 5.62 & 0.57 & 0.96 \\
\hline L.S.D. at $5 \%$ & 1.83 & 1.69 & 0.39 & 0.25 & 0.27 & 0.05 & 1.16 & 0.24 & 0.02 & 0.05 \\
\hline
\end{tabular}

faba bean could be manipulating by application of of FA and/or cultivars (Table 6).

\section{Conclusions}

In conclusion, FA can be easily applied to sustainable faba bean production in the field. Application of FA significantly increased every morphological attribute of four faba bean cultivars, including seed and straw yield, the main consumer-related traits. FA at $9 \mathrm{gL}^{-1}$ and Sakha 4 cultivar, improves growth performance, yield and shows the greatest potential for producing legume seeds with an enhanced nutritional quality for uses as food and feed (by increasing the level of carbohydrates, proteins, minerals, Lys, Trp, and Phe). 


\section{Acknowledgements}

This study was supported by botany department, National Research Centre, Egypt.

\section{Funding}

Not applicable

\section{Availability of data and materials}

The authors agree to use data and materials with refer to the reference

\section{Authors' contributions}

All authors participated in preparing and treating the experiments and writing the manuscript. All authors read and approved the final manuscript.

\section{Ethics approval and consent to participate}

Not applicable

\section{Consent for publication}

The authors are consent for publication this manuscript in the NRC bulletin

\section{Competing interests}

The authors declare that they have no competing interests.

\section{Publisher's Note}

Springer Nature remains neutral with regard to jurisdictional claims in published maps and institutional affiliations.

\section{Author details}

'Department of Botany, National Research Centre, El Buhouth St., Dokki, Cairo 12622, Egypt. ${ }^{2}$ Department of Botany and Microbiology, Faculty of Science, Helwan University, Cairo, Egypt.

\section{Received: 25 August 2018 Accepted: 11 December 2018}

Published online: 05 January 2019

\section{References}

A.O.A.C (1988) Official methods of analysis, 21 st edn. Association of Official Analytical Chemists, Washington, D.C.

Abdel-Gawad AA, El-Shouny KA, Saleh SA, Ahmed MA (1980) The relation between the efficiency of leaf surface and the growth of some wheat cultivars. Egypt Res Bull 1412:1-17

Aminifard MH, Aroiee H, Nemati H, Azizi M, Jaafar HZE (2012) Fulvic acid affects pepper antioxidant activity and fruit quality. Afr J Biotech 11:13179-13185

Anjum SA, Wang L, Farooq M, Xue L, Ali S (2011) Fulvic acid application improves the maize performance under well watered and drought conditions. J Agron Crop Sci 197:409-417

Asp H, Berggren D (1990) Phosphate and calcium uptake in beech (Fagus sylvatica) in the presence of aluminium and natural fulvic acids. Physiol Plant 80:307-314

Berbara RLL, García AC (2014) Humic substances and plant defense metabolism. In: Ahmad P, Wani MR (eds) Physiological mechanisms and adaptation strategies in plants under changing enviornoment: volume 1. Springer Science+Business Media, New York, pp 297-319

Bocanegra MP, Lobartini JC, Orioli GA (2006) Plant uptake of iron chelated by humic acids of different molecular weights. Commun Soil Sci Plant Anal 37:1-2

Brown JD, Lilliland O (1946) Rapid determination of $\mathrm{K}$ and $\mathrm{Na}$ in plant material and soil extracts by flame photometer. J Am Soc Hortic Sci 48:341-346

Bulgari R, Cocetta G, Trivellini A, Vernieri P, Ferrante A (2015) Biostimulants and crop responses: a review. Biol Agric Hortic 31(1):1-17

Calvo P, Nelson L, Kloepper JW (2014) Agricultural uses of plant biostimulants. Plant Soil 383:3-41

Carpena R, Ramon AH, Garate A (1990) The effects of short term deficiency of B, K, $\mathrm{Ca}$, and $\mathrm{Mg}$ ions distribution in leaves and roots of tomato (Lycopresicon esculentem L.) plants. In: Van Bensichem ML (ed) Plant Nutr Physio Appl, pp 287-290

Cazzato E, Tufarelli V, Ceci E, Stellacci AM, Laudadio V (2012) Quality, yield and nitrogen fixation of faba bean seeds as affected by sulphur fertilization. Acta Agri Scan Section B - Soil Plant Sci 62(8):732-738

Chaieb V, González JL, López-Mesas M, Bouslama M, Valiente M (2011) Polyphenols content and antioxidant capacity of thirteen faba bean (Vicia faba L.) genotypes cultivated in Tunisia. Food Res Int 44(4):970-977
Chapman HD, Pratt PF. Methods of analysis for soil, plant and water. Agric Sci Dept Univ of California USA 309. Chem 1978;36:345-347

Chen Y, Clapp CE, Magen H (2004) Mechanisms of plant growth stimulation by humic substances: the role of organo-iron complexes. Soil Sci Plant Nutr 50: 1089-1095

Çimrin KM, Türkmen Ö, Turan M, Tuncer B (2010) Phosphorus and humic acid application alleviate salinity stress of pepper seedling. Afr J Biotec 9(36):5845-5851

Dobbss LB, Medici LO, Peres LEP, Pino-Nunes LE, Rumjanek VM, Façanha AR, Canellas LP (2007) Changes in root development of Arabidopsis promoted by organic matter from oxisosls. Ann Appl Biol 151:199-211

Dubois M, Gilles KA, Hamilton JR, Robers PA, Smith F (1956) Colorimetric method for determination of sugar and related substances. An Chem 28:350-356

Eyheraguibel B, Silvestre J, Morard P (2008) Effects of humic substances derived from organic waste enhancement on the growth and mineral nutrition of maize. Bioresour Technol 99:4206-4212

Flore HE, Filner P (1985) Metabolic relationships of putrescine, GABA and alkaloids in cell and root cultures of solanaceae. In: Nenmann KH, Barz W, Reinhard E (eds) Primary and secondary metabolism of plant cell cultures. Springer-Verlag, Berlin, pp 174-185

Galston AW, Sawhney RK (1990) Polyamines in plant physiology. Plant Physi 94:406-410

Kumar A, Nidhi NP, Sinha SK (2015) Nutritional and antinutritional attributes of faba bean (Vicia faba L.) germplasms growing in Bihar, India. Physiol Mol Biol Plants 21(1):159-162

Lichtenthaler HK, Wellburn AR (1983) Determination of total carotenoids and chlorophylls $a$ and $b$ of leaf extracts in different solvents. Biochem Soc Trans 11:591-592

MacDonald MJ, D'Cunha GB (2007) A modern view of phenylalanine ammonia lyase. Biochem Cell Biol 85:273-282

Murillo JM, Madejón E, Madejón P, Cabrera F (2005) The response of wild olive to the addition of a fulvic acid-rich amendment to soils polluted by trace elements (SW Spain). J Arid Environ 63:284-303

Nardi S, Muscolo A, Vaccaro S, Baiano S, Spaccini R, Piccolo A (2007) Relationship between molecular characteristics of soil humic fractions and glycolytic pathway and Krebs cycle in maize seedlings. Soil Biol Biochem 39:3138-3146

Olivares FL, Aguiar NO, Rosa RCC, Canellas LP (2015) Substrate biofortification in combination with foliar sprays of plant growth promoting bacteria and humic substances boosts production of organic tomatoes. Sci Hortic 183:100-108

Plummer DT (1978) An introduction to practical biochemistry, 2nd edn. McGraw-Hill Book Company (U.K.) Ltd., London, p 368

Radford PJ (1967) Growth analysis formulae - their use and abuse 1. Crop Sci 7:171-175

Radwanski ER, Last RL (1995) Tryptophan biosynthesis and metabolism: biochemical and molecular genetics. Plant Cell 7:921-934

Rauthan BS, Schnitzer M (1981) Effects of a soil fulvic acid on the growth and nutrient content of cucumber (Cucumis sativus) plants. Plant Soil 63:491-495

Rose MT, Patti AF, Little KR, Brown AL, Jackson WR, Cavagnaro TR (2014) A metaanalysis and review of plant-growth response to humic substances: practical implications for agriculture D.S. Sparks (Ed.). Ad Agro 124:37-89

Sánchez-Sánchez A, Sánchez-Andreu J, Juárez M, Jordá J, Bermúdez D (2002) Humic substances and amino acids improve effectiveness of chelate Fe EDDHA in lemon trees. J Plant Nutr 25:2433-2442

Schiavon M, Pizzeghello D, Muscolo A, Vaccaro S, Francioso O, Nardi S (2010) High molecular size humic substances enhance phenylpropanoid metabolism in maize (Zea mays L.). J Chem Ecol 36:662-669

Sestak Z, Catsky J, Jarvis PG (1971) In: WNV J (ed) Plant photosynthetic production, manual of methods. Publications, The Hungus, pp 343-381

Snedecor GW, Cochran WG (1990) Statistical methods, 8th edn. lowa State University Press, Ames, p 609

Ufaz S, Galili G (2008) Improving the content of essential amino acids in crop plants: goals and opportunities. Am Soci Plant Biolo 147(3):954-961

Van Oosten MJ, Pepe O, De Pascale S, Silletti S, Maggio A (2017) Review: the role of biostimulants and bioeffectors as alleviators of abiotic stress in crop plants. Chemical and Biological Technologies in Agriculture 4:5. https://doi.org/10. 1186/s40538-017-0089-5

Vivekanadan AS, Gunasena HPM, Sivanayagam T (1972) Statistical evaluation of the accuracy of three techniques used in the estimation of leaf area of crop plants. Indian J Agri Sci 42:857-860

Walden R, Cordeiro A, Tiburcio AF (1997) Polyamines: small molecules triggering pathways in growth and development. Plant Physiol 113:1009-1013

Yakhin O, Lubyanov AA, Yakhin IA, Brown P (2017) Biostimulants in plant science: a global perspective. Front Plant Sci 7:2049. https://doi.org/10.3389/fpls. 2016.02049 
Zancani M, Bertolini A, Petrussa E, Krajñájivá J, Piccolo A (2011) Fulvic acid affects proliferation and maturation phases in Abies cephalonica embryogenic cells. J Plant Physiol 168:1226-1233

Zimmerli L, Hou BH, Tsai CH, Jakab G, Mauch-Mani B, Somerville S (2008) The

xenobiotic beta-aminobutyric acid enhances Arabidopsis thermotolerance. Plant J 53:144-156

Submit your manuscript to a SpringerOpen ${ }^{\mathcal{O}}$ journal and benefit from:

- Convenient online submission

- Rigorous peer review

- Open access: articles freely available online

- High visibility within the field

- Retaining the copyright to your article

Submit your next manuscript at $\boldsymbol{\sim}$ springeropen.com 